\title{
Perception of movement extent depends on the extent of previous movements
}

\author{
Tatjana Seizova-Cajic ${ }^{2}$, Janette L Smith ${ }^{3}$, Janet L Taylor ${ }^{1}$ and Simon C Gandevia ${ }^{1}$ \\ ${ }^{1}$ Prince of Wales Medical Research Institute and University of New South Wales \\ ${ }^{2}$ Faculty of Health Sciences, University of Sydney \\ ${ }^{3}$ School of Psychology, University of Newcastle
}

\section{Corresponding author:}

Dr Tatjana Seizova-Cajic

Faculty of Health Sciences

University of Sydney

Lidcombe, NSW 1825

Phone: 61293519190

Email: t.seizovacajic@usyd.edu.au

Keywords: Proprioception, kinesthesis, adaptation, aftereffects, movement extent 


\begin{abstract}
We report an aftereffect in perception of the extent (or degree or range) of joint movement, showing for the first time that a prolonged exposure to a passive back-andforth movement of a certain extent results in a change in judgment of the extent of a subsequently presented movement. The adapting stimulus, movement about the wrist, had an extent of either $30^{\circ}$ or $75^{\circ}$, while the test stimulus was a $50^{\circ}$ movement. Following a 4-min adaptation period, the estimated magnitudes of the test stimuli were $61^{\circ}$ and $36^{\circ}$ in the $30^{\circ}$ and $75^{\circ}$ condition, respectively (t-test(6) $=9.6 ; \mathrm{p}<0.001$ ). The observed effect is an instance of repulsion or contrast commonly described in perception literature, with perceived value of the test stimulus pushed away from the adapting stimulus.
\end{abstract}




\section{Introduction}

Adaptation is a ubiquitous phenomenon in sensory processing. It encompasses a range of neurophysiological and phenomenological changes that reveal dependence of the current state of a sensory system on the history of stimulation. Adaptation has been most studied in vision. Temporal context (or history of stimulation) that results in adaptation has been likened to the spatial context, because both strongly affect perception of visual stimuli and, at least for some perceptual features, in similar ways (see Clifford 2002; Schwartz, Hsu and Dayan 2007, Figure 2). Context-dependence is a desirable feature of a sensory system because context constrains the 'meaning' of sensory input, making some possibilities more likely than others. Furthermore, the stable, dominant or redundant contextual features provide a reference against which other stimuli can be economically coded. These are some of the considerations that gave rise to theories of adaptation that emphasize its function (see, e.g., Attneave 1954; Barlow 1990; Clifford and Rhodes 2005; Helson 1948) rather than seeing it simply as fatigue or an unfortunate by-product of the nature of sensory processing. In vision, adaptation is also a tool to investigate the mechanisms of perceptual coding.

Less is known about adaptation in basic mechanisms of proprioception, and adaptation has rarely been used as a tool to probe proprioceptive mechanisms. Proprioception encompasses sense of position, movement, heaviness and effort (Matthews 1982; McCloskey 1978; Gandevia 1996). The temporal context in which it operates, which determines the adaptation state of the proprioceptive system, consists of previous positions and movements of body part(s), its relationship with vision, and history of exposure to specific force-fields, including gravity (for discussion of adaptation to visual distortions, see Welch 1978; for adaptation to altered force-fields, see DiZio and Lackner 2002). The present study is concerned with adaptation in conscious perception of movement based on proprioception, in particular, in perception of the extent (or range or degree) of passive movement.

Our goal is to establish whether repetitive bidirectional movement of a certain extent results in a) an adaptation, or phenomenological change in the adapting stimulus, such that its perceived extent changes, and b) an aftereffect, or changes in perception of 
subsequent movements of different extent in comparison to their perception in the nonadapted state.

\section{Methods}

The experimenter imposed repetitive flexion-extension movements on the wrist of the relaxed arm of the participant. The task was to estimate verbally the angle through which the wrist was being rotated when prompted (every $40 \mathrm{~s}$ ). The angular extent of the adapting movement was either $30^{\circ}$ or $75^{\circ}$. The extent of the test movement was always $50^{\circ}$.

\section{Participants}

Seven volunteers from staff at the POWMRI with no known sensory or motor impairments participated in the study (4 females). The Human Research Ethics Committees of the University of New South Wales and University of Sydney approved the study.

\section{Design}

There were two experimental conditions and one control condition in a repeatedmeasures design, with each condition presented once. The two experimental conditions differed in the extent of the adapting movement, which was either $30^{\circ}$ (Adapt $30^{\circ}$ condition) or $75^{\circ}$ (Adapt $75^{\circ}$ condition). The movement used to test the aftereffect (the test stimulus) was $50^{\circ}$ in both cases. The adapting movement was presented throughout the run to maintain the adaptation (with a total presentation time of $776 \mathrm{~s}$ ), interrupted by brief presentations of the test stimulus (for a total of $64 \mathrm{~s}$ ). For the first $4 \mathrm{~min}$ of the Adapt conditions, the participant was exposed to adapting movement only, and judged its extent every 40s, but after that time, the test movement and adapting movement were judged alternately. The Control condition used $30^{\circ}, 50^{\circ}$ and $75^{\circ}$ movements for equal durations to determine the baseline values in judgment of these extents. The order of conditions was counterbalanced across participants. 


\section{Stimuli and apparatus}

Participants were seated with the right forearm strapped to a table and hand held in a manipulandum, which could be rotated in the flexion-extension plane coaxial with the wrist. The fingers were clamped in full extension. The wrist was deemed to be at $0^{\circ}$ when the metacarpals were in line with the forearm, and all movements were performed symmetrically around that position. The forearm, hand and apparatus were covered with a cloth and the participant also closed her/his eyes. The experimenter manually flexed and extended the wrist to different positions marked on the table.

The average speed of the back-and-forth movement was $\sim 50 \%$, following the rhythm of a pre-recorded metronome presented to the experimenter through headphones. Three different beats were used and at each beat, the hand reached one of the two extreme positions of the required movement extent $\left(30^{\circ}, 50^{\circ}\right.$ or $\left.75^{\circ}\right)$, maintaining the same average required speed in each case. The movement never stopped during the run; following pre-recorded instructions, the experimenter made transitions from one to another required movement extent without a pause.

The adapting movement was presented for $93 \%$ of the time, and the test movement for $7 \%$ of the time in the Adapt runs. In the control run, each of the three stimuli were presented for equal proportions of the time (33\%). Details of the temporal structure of each run were as follows:

Adapt $30^{\circ}$ condition: $30^{\circ}$ for 240 s, then 14 cycles, each of $50^{\circ}$ for 8 s followed by $30^{\circ}$ for $72 \mathrm{~s} ;$

Adapt $75^{\circ}$ condition: $75^{\circ}$ for 240 s, then 14 cycles, each of $50^{\circ}$ for 8 s followed by $75^{\circ}$ for $72 \mathrm{~s}$

Control condition: $30^{\circ}, 50^{\circ}$ and $75^{\circ}$ extents were presented in quasi-random order (the same for all the participants) for 40s each.

To ensure the subjects remained relaxed throughout the experiment, electromyographic activity (EMG) was recorded with surface electrodes over flexor carpi radialis and extensor carpi radialis (filtered at 16-1000 Hz; CED 1902 amplifiers, Cambridge Electronic Design, Cambridge, UK). 
A potentiometer coaxial with the wrist (mounted underneath the table) signaled the extent and speed of hand movement. Angular position and EMG signals were recorded to computer through a laboratory interface (CED 1401 with Spike2 software) The intended speed of movement in all the conditions was $50 \%$, and actual speed ranged from $50 \%$ s$54 \%$, with the larger movements being slightly faster (Table 1 ). The recorded values for the extent of movement were as intended for $30^{\circ}$ and $50^{\circ}$ movements, and $77^{\circ}$ for the $75^{\circ}$ movement. Note that for the critical, $50^{\circ}$ movement, both speed and extent were nearly identical across the three conditions.

Table 1. Angular speed and extent of hand rotation around the wrist (means and standard deviations), potentiometer readings.

\begin{tabular}{|ccc|}
\hline & Speed (deg/s) & Extent (deg) \\
\hline Adapt $30^{\circ}, 30^{\circ}$ movement & $50.8(4.4)$ & $29.6(1.3)$ \\
Adapt $30^{\circ}, 50^{\circ}$ movement & $52.5(4.8)$ & $49.9(1.1)$ \\
\hline Adapt $75^{\circ}, 50^{\circ}$ movement & $52.3(4.4)$ & $49.9(1.2)$ \\
Adapt $75^{\circ}, 75^{\circ}$ movement & $53.7(4.1)$ & $77.2(1.3)$ \\
\hline Control, $30^{\circ}$ movement & $51.8(4.4)$ & $29.7(1.3)$ \\
Control, $50^{\circ}$ movement & $52.0(4.4)$ & $50.1(1.2)$ \\
Control, $75^{\circ}$ movement & $53.9(4.2)$ & $77.3(1.2)$ \\
\hline
\end{tabular}

\section{Procedure}

Participants received instructions to estimate, in degrees, the angular excursion of the hand from one to the other endpoint of the repetitive movement. The experimenter moved the manipulandum back-and-forth a few times to familiarize the subjects with the range and repetitive nature of movement. Subjects were instructed to relax if EMG activity was detected. The three experimental runs (Adapt $30^{\circ}$, Adapt $75^{\circ}$ and Control) lasted 14 min each, with breaks of at least $5 \mathrm{~min}$ in between. They were preceded by a practice run (without feedback) consisting of the first three stimuli from the control condition.

Every $40 \mathrm{~s}$ during each run (starting $40 \mathrm{~s}$ after the beginning of the run), the participant was prompted to estimate magnitude of the angular extent of the movement. Recall that in Adapt $30^{\circ}$ and Adapt $75^{\circ}$ conditions, the $50^{\circ}$ test movement was only presented for $8 \mathrm{~s}$ at a time (to minimize the change in any adaptation). The magnitude estimate for this 
stimulus was requested $4 \mathrm{~s}$ into this $8 \mathrm{~s}$ period (after two consecutive $50^{\circ}$ back-and-forth movements). Participants always responded before the movement they judged was completed. In the Control run, each stimulus was presented for 40s, and the estimate was required approximately $4 \mathrm{~s}$ before the completion of the presentation.

\section{Data analysis}

Raw data were magnitude estimates of the angular extent of hand movement, both for the adapting and for the test stimulus. Absolute accuracy of the numerical judgments is not of interest, but only differences across conditions and any changes occurring during the run. We consider two aspects of the results: trends in the responses as a function of time, and the aftereffect of continuous movement, that is, the difference in perception of the $50^{\circ}$ movement following the adaptation to $30^{\circ}$ as compared to $75^{\circ}$.

\section{Results}

Figure 1A-C shows the group mean responses in the three experimental conditions as a function of time from the onset of stimulation. In Adapt $30^{\circ}$ condition (Figure 1A), responses to the $30^{\circ}$ adapting movement were rather stable throughout the run, while the $50^{\circ}$ test movement had a decreasing trend as the run progressed (the linear trend was significant, with F $((1,6)=6.710 ; \mathrm{p}<0.05)$. In Adapt $75^{\circ}$ condition (Figure 1B), responses to the $75^{\circ}$ adapting movement decreased $(\mathrm{F}(1,6)=6.657 ; \mathrm{p}<0.05)$, while the responses to the $50^{\circ}$ test movement were stable. In the Control run (Figure $1 \mathrm{C}$ ), the responses to the $75^{\circ}$ stimulus, but not the others, also gradually decreased $(\mathrm{F}=6.324 ; \mathrm{p}<0.05)$. Thus the largest stimulus in each run showed a decreasing response.

Table 2 shows estimates of movement extents in all three conditions, averaged over time. The critical finding is that the $50^{\circ}$ movement angle was judged on average as $\sim 25^{\circ}$ greater in Adapt $30^{\circ}$ than in Adapt $75^{\circ}$ condition (95\% confidence interval $18.9^{\circ}-31.9^{\circ}$ ). This difference between the means was significant at the 0.001 level (repeated measures t-test $(6)=9.57)$. Unlike the $50^{\circ}$ movement, $30^{\circ}$ and $75^{\circ}$ movements were judged as similar in different conditions ( $p$ values for paired t-tests for difference between the means were $>0.05)$. 
In the Control condition, the value of the estimate for the $50^{\circ}$ movement was approximately midway between the estimates of the same movement extent in the Adapt conditions. In this condition, the $50^{\circ}$ movement was presented before any other stimulus and then three times following the $35^{\circ}$ movement, and three times following the $75^{\circ}$ movement, but previous stimulation did not seem to influence the estimates. This can be seen in Figure 1C, which shows the stimuli in the order in which they were presented to all the participants.

Table 2. Estimates of angular extent of movement (means and standard errors of the mean for 7 participants). Magnitude estimates of the critical, $50^{\circ}$ movement extent are in bold; note that it was judged differently in different conditions.

\begin{tabular}{|cc|}
\hline & Estimated extent (deg) \\
\hline Adapt $30^{\circ}, 30^{\circ}$ movement & $33.0(3.3)$ \\
Adapt $30^{\circ}, 50^{\circ}$ movement & $\mathbf{6 1 . 4}(7.3)$ \\
\hline Adapt $75^{\circ}, 50^{\circ}$ movement & $\mathbf{3 6 . 0}(6.4)$ \\
Adapt $75^{\circ}, 75^{\circ}$ movement & $67.5(11.0)$ \\
\hline Control, $30^{\circ}$ movement & $27.9(4.4)$ \\
Control, $50^{\circ}$ movement & $\mathbf{4 8 . 0}(9.3)$ \\
Control, $75^{\circ}$ movement & $70.4(10.6)$ \\
\hline
\end{tabular}

\section{Discussion}

We asked first, whether the perception of the adapting movement changes over time, and, second, whether prolonged movement results in an aftereffect on movements of a different extent than the adapting movement. There is no clear evidence that perception of the adapting movement extent itself changes during the 14 min period. The response to the $75^{\circ}$ adapting stimulus did gradually decrease, but this did not happen with the smaller, $30^{\circ}$ adapting stimulus. In fact, responses to the largest movements in all conditions decreased as the run progressed, resulting in compression of the range of responses. Second, there is a clear aftereffect, which can be described as a repulsion: movements greater than the adapting movement were overestimated in comparison to the non-adapted state, and movements smaller than the adapting movement were underestimated. In other words, the perceived value of the test stimulus deviated away 
from the adapting stimulus. The effect is large, with estimates of the same movement differing on average by a factor of 1.7 depending on previous stimulation.

The time course of adaptation has not been revealed. We presented test stimuli different from the adapting stimulus after 4 min of adaptation in the Adapt conditions, and observed aftereffects, but it is possible that they were measurable after a much shorter time period. Less than a minute of adaptation is sufficient for colour and motion aftereffects, and even much shorter exposures, (less than a second), result in aftereffects (e.g., Wolfe, 1984). In the Control condition, different extents were presented for $40 \mathrm{~s}$ each, one after the other, and judged towards the end of the presentation period, after 36s. This appears to be sufficient time for any effects of the previous stimulation to dissipate so that judgment for the $50^{\circ}$ movement was the same (and close to $50^{\circ}$ ) whether preceded by no movement or by a $40 \mathrm{~s}$ period of the $35^{\circ}$ or $70^{\circ}$ stimulus.

Repulsive aftereffects are "a psychophysical signature of central-tendency coding" (Suzuki 2005, p 140), in which a feature is encoded by the combined output of a population of neural units with systematically different but overlapping tuning. According to a widely-held view, aftereffects occur as a consequence of a decreased resting level and responsiveness in the neurons excited by the adapting stimulus, such that other, less adapted and non-adapted neurons have a relatively greater contribution to the combined output (e.g., Mather and Harris 1998). This results in a shift of the peak of activity in the population of neurons, and a change in perception of subsequently presented stimuli. The functional significance of repulsive shifts is supposed to be an improved discrimination for the stimuli likely to occur at a given adaptation level.

What tuning properties of neurons could underlie adaptation in the present study? Clearly, neurons would need to be involved in the perception of extent of movement, but would not necessarily be labeled lines with different preferred extents of movement. Adaptation could also occur at the sites that encode position or movement because they both may contribute to perceived extent. Position and movement are coded relatively independently from the periphery to the somatosensory cortex of primates (e.g. Mountcastle and Powell 1959; Gardner and Costanzo 1981). Therefore, adaptation could 
potentially occur in at least three different kinds of neural structures: neurons tuned to position, neurons tuned to speed and/or direction of movement, and neurons tuned to the extent itself, irrespective of position or speed and direction of movement. These possibilities are not mutually exclusive, and further experiments are needed to tease them apart. If, for example, the extent aftereffect shows position invariance, then purely position channels do not play a key role in adaptation to extent. An experiment for testing position invariance would use movements centered on different midpoints in the adaptation and test phases; an undiminished aftereffect would show that there is position invariance. Speed invariance can be tested in an analogous manner.

Perception of extent of the adapting movement showed no clear change due to adaptation, yet, given that aftereffects occurred, we propose a change in the responsiveness at a neural level. This is consistent with the model of coding by place in which the adapted channel becomes increasingly less responsive, but its activity is still at levels greater than in other channels. Being a 'winner', or the site of peak activity, this channel continues to signal the same movement extent for as long as the same stimulus is presented, even though its firing rate has decreased. Presentation of a different stimulus (a test extent larger or smaller than the adapting extent), however, reveals that responsiveness in the population of neurons has changed, resulting in the aftereffect. Similar findings are obtained in visual adaptation to motion. After observers adapt to a pattern moving in a given direction, they are shown other test directions. Then, they exhibit a clear repulsive aftereffect, but perception of the adapting direction itself does not change (e.g. Schrater and Simoncelli, 1998, Figure 1). As spatial or extensive properties in both vision and proprioception appear to behave similarly during adaptation, they may have similar coding schemes.

The intensive property, speed, behaves differently in vision, and possibly also in proprioception. In vision, a pattern moving at the constant speed appears to gradually slow down (Thompson, 1982). Perhaps in our study the perceived speed of movement, which we did not measure, also gradually decreased during adaptation. This would be expected of a perceptual attribute coded by frequency, which presumably decreases during adaptation. 
The present results show that (temporal) context affects judgments of stimulus magnitude in proprioception. We interpreted this as adaptation, or a true change in perception. Is there an equally plausible interpretation in terms of post-perceptual processes, usually labeled the 'response bias' (see Poulton 1979)? According to the latter interpretation, the critical, $50^{\circ}$ movement, was not perceived differently in different experimental conditions; it was only judged differently. A post-perceptual mechanism, in which sensory processing through to perception is unchanged but the perception evokes a different response, is implied here (for a discussion of these opposing views see, e.g., Gescheider, 1997, pp 255-263). We favour the perceptual, adaptation explanation because it is theoretically linked to neural mechanisms and our findings are consistent with a large literature in vision, audition and other modalities, which describes similar 'contextual' effects that cannot be explained by response bias. These include effects that (i) are undeniably perceptual in nature (e.g. the classical visual colour aftereffect: following prolonged exposure to red, the mixture of wavelengths normally perceived as white appears green), (ii) use discrimination judgments rather than ratings, and (iii) are accompanied by decreased responsiveness of sensory cells tuned to the adapting stimulus.

Finally, our findings complement other studies concerned with perceptual adaptation to basic properties of limb movement (see Gibson 1933, for adaptation to the curvature or shape of movement trajectory; Kito et al 2006 and Seizova-Cajic et al 2007, for adaptation to movement velocity). These investigations clearly show that temporal context is as important in proprioception as it is in vision, presumably for similar, functional reasons. However, at this stage, research in proprioception has a long way to go before we have enough knowledge to propose meaningful models of the neural coding of movement and the role of adaptation.

\section{Acknowledgments}

This work was supported by funding from the National Health and Medical Research Council of Australia to JT and SC. 


\section{References}

1. Attneave F (1954) Informational aspects of visual perception. Psych Rev 61:183193.

2. Barlow H (1990) A theory about the functional role and synaptic mechanism of visual after-effects. In: Blakemore C (ed) Vision: coding and efficiency Cambridge University Press. pp 363-375.

3. Clifford C (2002) Perceptual adaptation: motion parallels orientation, Trends in Cogn Sci 6:136-143.

4. Clifford C and Rhodes G (2005) Fitting the Mind to the World: Adaptation and After-Effects in High-Level Vision, Oxford University Press. 376 p.

5. DiZio P and Lackner J (2002) Proprioceptive adaptation and aftereffects. In Stanney K (ed): Handbook of Virtual Environments. New York: Lawrence Erlbaum Associates. pp 751-777.

6. Gandevia S (1996) Kinesthesia: roles for afferent signals and motor commands. In: Rowell L and Shepherd J (eds) Handbook on Integration of Motor, Circulatory, Respiratory and Metabolic Control During Exercise pp. 128-172. American Physiological Society.

7. Gardner and Costanzo (1981) Properties of kinaesthetic neurons in somatosensory cortex of awake monkeys. Brain Res 214:301-319.

8. Gescheider, G (1997) Psychophysics: The Fundamentals (3 ${ }^{\text {rd }}$ edition). Lawrence Erlbaum Associates, London.

9. Gibson J (1933) Adaptation, aftereffect, and contrast in the perception of curved lines. Jr Exp Psych 16:1-31.

10. Helson H (1948) Adaptation-level as a basis for a quantitative theory of frames of reference. Psych Rev 55:297-313.

11. Kito T, Hashimoto T, Yoneda T, Katamoto S and Naito E (2006) Sensory processing during kinesthetic aftereffect following illusory hand movement elicited by tendon vibration. Brain Res 1114:75-84.

12. Mather $G$ and Harris J (1998) Theoretical models of the motion aftereffect. In: Mather G, Verstraten F and Anstis S, editors. The motion aftereffect. Bradford: MIT Press. pp 157-185. 
13. Matthews PBC (1982) Where does Sherrington's 'muscular sense' originate? Muscles, joints, corollary discharges? Ann Rev Neurosci 5:189-218.

14. McCloskey D (1978) Kinesthetic sensibility. Physiol Rev 58 (4):763-820.

15. Mountcastle VB and Powell TPS (1959) Central nervous mechanisms subserving position sense and kinesthesis. Johns Hopkins Hosp Bull 105:173-200.

16. Poulton E 1979: Models for biases in judging sensory magnitude. Psych Bul 86, 4:777-803.

17. Schrater P \& Simoncelli P (1998) Local velocity representation: evidence from motion adaptation Vision Research 38:3899-3912.

18. Schwartz O, Hsu A and Dayan P (2007) Space and time in visual context. Nat Rev Neurosci 8:522-535.

19. Seizova-Cajic T, Smith J, Taylor J \& Gandevia S (2007): Propriceptive movement illusions due to prolonged stimulation: reversals and aftereffects. PLoS ONE 2(10).

20. Suzuki S (2005) High-level pattern coding revealed by brief shape aftereffects. In C. Clifford and G. Rhodes (eds) Fitting the Mind to the World: Adaptation and After-Effects in High-Level Vision, Oxford University Press.

21. Thompson P (1980) Velocity after-effects: the effects of adaptation to moving stimuli on the perception of subsequently seen moving stimuli. Vis Res 21:33745.

22. Welch RB (1978) Perceptual Modification. New York: Academic Press.

23. Wolfe JM (1984) Short test flashes produce large tilt aftereffects. Vis Res 24: 1959-1964. 
Figure 1. Group mean estimates for movements of different extents as a function of time ( $n=7$; error bars represent standard error of the mean). The dashed and dotted lines indicate true values of imposed movements in a given condition, indicated by the level where they meet the y-axis. A. Adapt $30^{\circ}$ condition. Circles and crosses: estimates of the $30^{\circ}$ and $50^{\circ}$ movements, respectively. B. Adapt $75^{\circ}$ condition. Circles and crosses: estimates of the $75^{\circ}$ and $50^{\circ}$ movements, respectively. C. Control condition. Circles, crosses and squares: estimates of the $30^{\circ}, 50^{\circ}$ and $75^{\circ}$ movements, respectively. Note that in all three conditions (A, B and $\mathrm{C}$ ), the crosses represent estimates of the critical, $50^{\circ}$ movement, and that those estimates vary widely across conditions.

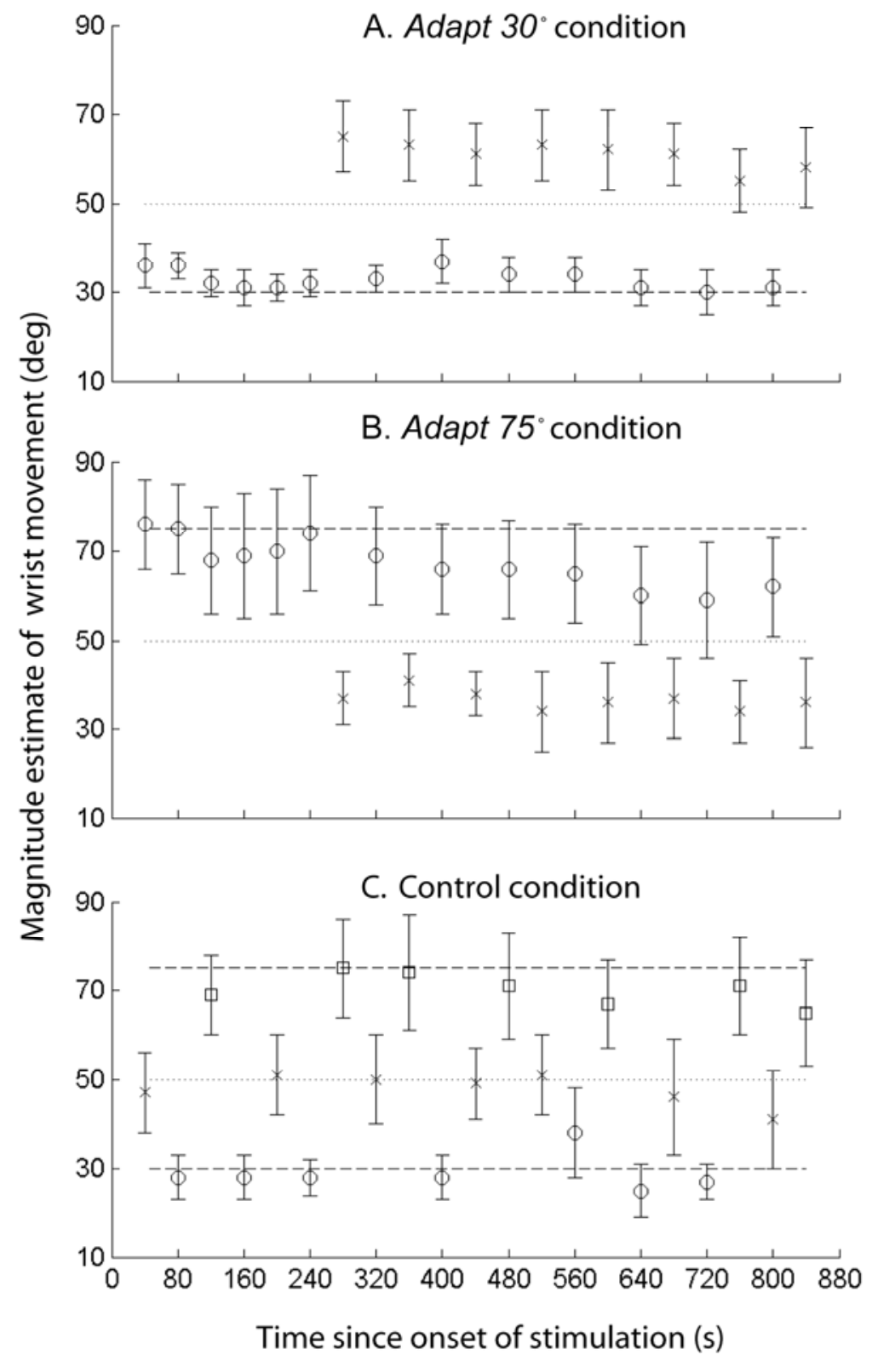

